\title{
Long-wavelength fast semiconductor saturable absorber mirrors using metamorphic growth on GaAs substrates
}

\author{
S. Suomalainen, A. Vainionpää, O. Tengvall, T. Hakulinen, S. Karirinne, \\ M. Guina, and O. G. Okhotnikov \\ Optoelectronics Research Centre, Tampere University of Technology, P.O. Box 692, 33101 Tampere, Finland
}

T. G. Euser and W. L. Vos

Complex Photonics Systems (COPS) and MESA + Research Institute, University of Twente, P.O. Box 217, 7500 AE Enschede, The Netherlands

(Received 25 April 2005; accepted 11 August 2005; published online 13 September 2005)

\begin{abstract}
Metamorphic growth of InP on GaAs has been used to decrease the absorption recovery time of $1.55 \mu \mathrm{m}$ semiconductor saturable absorber mirrors. We show that the recovery time can be reliably controlled by changing the thickness of an InP "lattice reformation layer" grown between the GaAs-based distributed Bragg reflector and the active region. Semiconductor saturable absorber mirrors with a thickness of the InP reformation layer around $200 \mathrm{~nm}$ or smaller exhibit a recovery time short enough to reliably mode-lock fiber lasers. (C) 2005 American Institute of Physics.
\end{abstract}

[DOI: $10.1063 / 1.2053364]$

Semiconductor saturable absorber mirrors (SESAMs) are nonlinear optical elements that have found applications in a large variety of fields ranging from passively modelocked lasers to all-optical regeneration and noise suppression in optical telecommunication links. ${ }^{1-3}$ SESAMs are particularly attractive for passive mode-locking of lasers. Ultrafast lasers passively mode-locked by SESAM exhibit reliable self-starting behavior, produce high-quality pulse trains, and are less complex and potentially cheaper than laser mode-locked by other techniques.

In order to provide efficient pulse shaping, the saturable absorption of a SESAM should recover to its initial value in a short time. For efficient and self-starting mode-locking, the recovery time should have values in the range from few picoseconds to few tens of picoseconds, depending on the properties of the gain medium and the laser cavity design. However, the recovery time for typical epitaxially grown compound semiconductors falls in the nanoseconds range. Therefore, the fabrication process of SESAMs should include special measures to reduce or control the recovery time. Another important aspect in controlling the absorber recovery time is that there is an optimal value for the absorber speed that leads to the best self-starting capability and stability of the passive mode-locking. ${ }^{4}$ The most common methods for reducing the absorption recovery time include low-temperature growth, ${ }^{5} \mathrm{Be}$ doping, ${ }^{6}$ proton bombardment, ${ }^{7}$ and ion irradiation. ${ }^{8}$ Each of these techniques brings in certain tradeoffs related to fabrication complexity, device reliability, degradation of nonlinearity, increased nonsaturable loss and accuracy of tuning the recovery time to desired values.

In this paper we present an alternative technique that offers a convenient way to decrease the absorption recovery time of the semiconductor saturable absorber. The method is based on controlling the crystalline quality of the absorbing region and thus the density of nonradiative recombination centers that are responsible for the fast response time of the absorption.

The general SESAM structure presented here starts with a distributed Bragg reflector (DBR), lattice-matched to sub- strate for achieving a low-loss reflectivity. The DBR is followed by a "lattice reformation" layer having a substantial difference of the lattice constant in respect to the DBR material and, consequently, to the substrate. Latticemismatching between the lattice reformation layer and the DBR is an instrumental to introduce nonradiative recombination centers caused by misfit and threading dislocations within the absorption region. Extremely high value of the dislocations density might result in high excess loss and deterioration of the SESAM's performance. Therefore, the density of recombination centers should be limited to the level required for achieving a certain value of the recovery time. To demonstrate the practicality of this method in reducing the absorption recovery time, we have fabricated a set of SESAM samples optimized for operation at long wavelength around $1.55 \mu \mathrm{m}$. The samples have been grown by all-solidsource molecular beam epitaxy on n-GaAs (100) substrates. As DBR we have used 25 pairs of AlAs/GaAs quarter-wave layers with a center wavelength of $1.55 \mu \mathrm{m}$. The active region of the absorber comprised five 11-nm-thick $\mathrm{In}_{0.53} \mathrm{Ga}_{0.47} \mathrm{As}$ quantum wells (QWs) with photoluminescence signal picked at $1.6 \mu \mathrm{m}$ and placed within a $3 / 2-\lambda$ cavity. An InP buffer layer was metamorphically grown between the last GaAs layer of the DBR and the active region. This layer is used to ensure the aforementioned lattice reformation and eventually allows the growth of $1.55 \mu \mathrm{m} \mathrm{InGaAs}$ QWs on nonlattice-matched GaAs substrate. The thickness of the InP-buffer layer is used to adjust the amount of dislocation within the absorption region.

Metamorphic growth of InP on GaAs has been used previously for fabrication of $\mathrm{p}-\mathrm{i}-\mathrm{n}$ photodiodes, ${ }^{9}$ heterojunction bipolar transistors, ${ }^{10}$ and monolithic growth of $1550 \mathrm{~nm}$ SESAMs on GaAs. ${ }^{11}$ In all these experiments the goal was to optimize the growth condition and the thickness of the InP buffer for enabling the fabrication of high-quality active layers on the top of it. This goal has been achieved by employing "multistep" epitaxy and relatively thick InP buffers. The growth of the InP buffer starts at low temperature $\left(\sim 400{ }^{\circ} \mathrm{C}\right)$ and increases gradually to normal growth tem- 


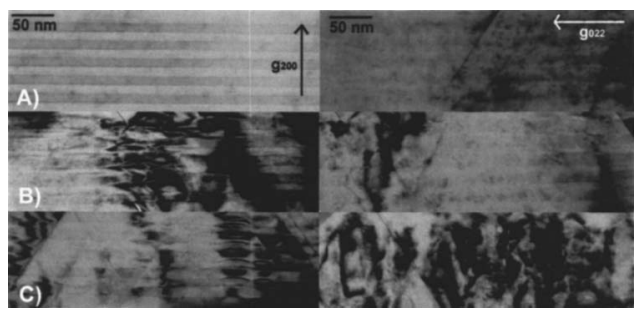

FIG. 1. TEM [200] and [022] bright field images from the quantum well area of sample A, B, and C.

perature resulting in a confinement of the dislocation at the interface between GaAs and InP.

In our experiment the aim was to generate a controllable density of defects within the active region. Therefore the InP-buffer layer was grown at normal temperature in a single epitaxy step. The thickness of InP buffer was varied and its influence on SESAM performance was investigated. Particularly, it was observed that the recovery time of the absorption increases with the thickness of the InP layer. This is due to the fact that crystalline quality of the QWs improves with an increase in the thickness of InP buffer. The crystallographic lattice perfection of the SESAMs was studied by crosssectional transmission electron microscope (TEM) using [200] and [022] bright field imaging. The TEM micrographs are shown in Fig. 1. Three samples with the thickness of InP-spacer layer of $439 \mathrm{~nm}$ (sample A), $208 \mathrm{~nm}$ (B), and 75 $\mathrm{nm}(\mathrm{C})$ were studied. One can see significant difference between samples A, B, and C. Particularly, in sample A there is almost no dislocation in the quantum well region, while in sample C the QW region is highly dislocated with the dislocations distributed nearly uniformly over quantum-well section. Sample B has a smaller number of dislocation in QWs compared to sample $\mathrm{C}$, owing to larger thickness of the InP reformation layer. The crystal quality of the SESAMs was further explored by high-resolution double-crystal x-ray diffraction (XRD). As it is expected, samples with thicker InP exhibited narrower full width at half-maximum and an increase in the peak intensities of the XRD spectrum indicating higher crystal quality owing to stronger lattice reformation. It should be noted here that the parameters of the QW absorption region were kept identical for all structures studied.

The effect of the thickness of the InP-spacer layer on the absorption recovery time was investigated by a standard degenerate pump-probe measurement setup. ${ }^{12}$ The optical pulses had a spectrum peaked at $1550 \mathrm{~nm}$. Cross-polarized pump and probe beams were incident normal to the SESAM surface. The average energy of the pump pulses was about $16 \mu \mathrm{J}$. The probe beam energy was less than $0.5 \%$ of the pump beam. Both beams were collimated to about $2.5 \mathrm{~mm}$ beam size. The recovery time constants derived from measurement using single-exponential fitting are 480, 150, and 40 ps for samples with thicknesses of InP layer of 439, 208, and $75 \mathrm{~nm}$, respectively. These data are plotted in Fig. 2 revealing an almost linear relation between the InP buffer thickness and the recovery time of the QW absorber. The data demonstrate a significant reduction in recovery time of absorption with a decrease in the thickness of InP buffer layer.

In order to prove that the fastest sample with a $75-\mathrm{nm}$ thin InP buffer, and therefore, with the highest density of defects, shows no degradation in nonlinear properties, we

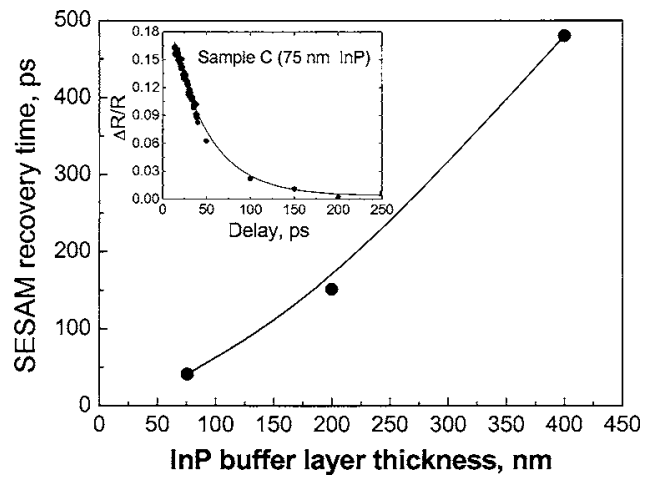

FIG. 2. Absorption recovery time against the thickness of InP lattice reformation layer. Inset: time resolved reflectivity response of SESAM samples with $75 \mathrm{~nm}$ InP lattice-reformation layer.

have measured the reflectivity variation versus pulse fluence of the probe signal. Figure 3 shows the nonlinear reflectivity curve for sample $\mathrm{C}$ at a wavelength of $1543 \mathrm{~nm}$. The experimental data were fitted numerically using a two-level saturable absorption model. ${ }^{4}$ The numerical fit gave a modulation depth of $\Delta R=0.15$ and a saturation fluence of $F_{\text {sat }}$ $=7.93 \mu \mathrm{J} / \mathrm{cm}^{2}$. Comparison with similar measurements made with samples A and B confirmed that nonlinear characteristics of the SESAM remain largely unchanged when the thickness of the InP buffer is small enough to ensure a significant decrease of the recovery time.

To examine their ability to start and stabilize passive mode-locking, the SESAMs were tested in a linear Er-doped fiber laser cavity defined by a fiber mirror at one end and the semiconductor saturable absorber mirror at the other end. The laser was core-pumped by a $110-\mathrm{mW} 980-\mathrm{nm}$ singlemode laser diode. It was found that SESAM with the recovery time of $480 \mathrm{ps}$ (sample A) could not start reliably a passive mode-locking operation, contrary to the SESAMs with fast absorption recovery (samples B and C). This feature is in agreement with our observations reported in Ref. 4. Moreover, when being mode-locked with a slow SESAM, the laser produced pulses with large pedestal. Using SESAMs with recovery times below 150 ps resulted in reliable self-starting mode-locked operation with pedestal-free pulses, as shown in Fig. 4. It can be also observed from this figure that faster recovery of the absorption provides shorter pulse durations.

In conclusion, we have demonstrated a long-wavelength semiconductor saturable absorber mirror by using metamorphic growth of InP on GaAs. The novel aspect of this work consists in the fact that the recovery time of absorption is

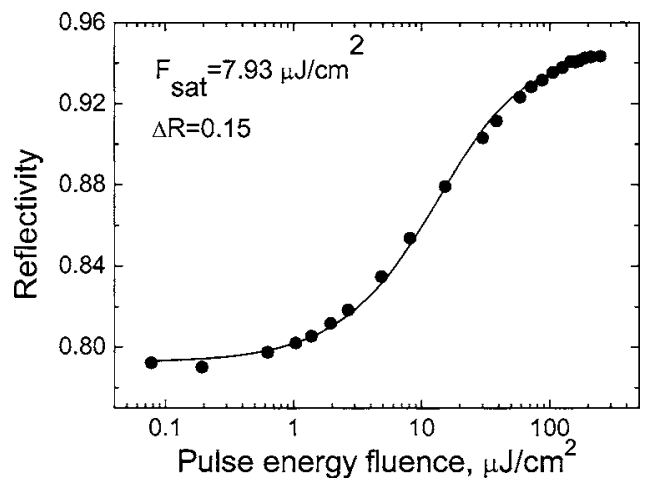

FIG. 3. Reflectivity as a function of the pulse energy fluence for sample C. 


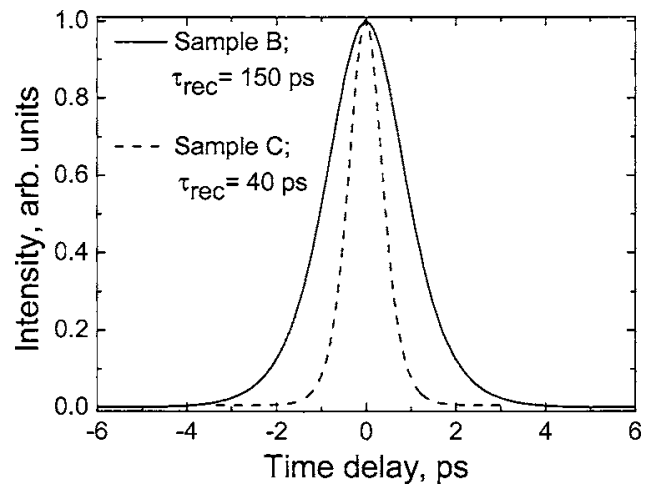

FIG. 4. Intensity autocorrelation traces for passive mode-locked operation obtained using SESAMs with recovery time of $150 \mathrm{ps}$ and $40 \mathrm{ps.}$

controlled by changing the thickness of the InP "lattice reformation layer" grown between the DBR and the active region. The mechanism underling this method to change the recovery time of the absorption is the lattice-mismatch induced defects within the active region. The method does not require any post-growth technological actions and has an advantage of flexibility in using semiconductor substrates, i.e., GaAs substrates can be used for long-wavelength operation. The performance of the SESAMs made using this technique has been illustrated in mode-locking fiber lasers. The control of the recovery time through lattice mismatch can be applied for a large variety of semiconductor material systems. Re- sults related to fabrication of fast SESAMs at $1060 \mathrm{~nm}$, with recovery time controlled through lattice mismatch management, will be reported elsewhere.

We gratefully acknowledge the support of the Academy of Finland within the framework of TULE-QUEST program and FOM/NOW.

${ }^{1}$ U. Keller, D. A. B. Miller, G. D. Boyd, T. H. Chiu, J. F. Ferguson, and M. T. Asom, Opt. Lett. 17, 505 (1992).

${ }^{2}$ A. Hirano, H. Kabayashi, H. Tsuda, R. Takahashi, M. Asobe, K. Sato, and K. Hagimoto, Electron. Lett. 34, 198 (1998).

${ }^{3}$ A. Isomäki, A.-M. Vainionpää, J. Lyytikäinen, and O. G. Okhotnikov, IEEE J. Quantum Electron. 39, 1481 (2003).

${ }^{4}$ R. Herda and O. G. Okhotnikov, Appl. Phys. Lett. 86, 011113 (2005).

${ }^{5}$ S. Gupta, J. F. Whitaker, and G. A. Mourou, IEEE J. Quantum Electron. 28, 2464 (1992).

${ }^{6}$ L. Qian, S. D. Benjamin, P. W. E. Smith, B. J. Robinson, and D. A. Thompson, Appl. Phys. Lett. 71, 1513 (1997).

${ }^{7}$ J. T. Gopinath, E. R. Thoen, E. M. Koontz, M. E. Grein, L. A. Kolodziejski, E. P. Ippen, and J. P. Donnelly, Proc. CLEO 698 (2001).

${ }^{8}$ E. L. Delpon, J. L. Oudar, N. Bouché, R. Raj, A. Shen, N. Stelmakh, and J. M. Lourtioz, Appl. Phys. Lett. 72, 759 (1998).

${ }^{9}$ A. G. Dentai, J. C. Campbell, C. H. Joyner, and G. J. Qua, Electron. Lett. 23, 38 (1987).

${ }^{10}$ H. Q. Zheng, K. Radhakrishnan, H. Wang, K. H. Yuan, S. F. Yoon, and G. I. Ng, Appl. Phys. Lett. 77, 869 (2000).

${ }^{11}$ B. C. Collings, K. Bergman, S. T. Cundiff, S. Tsuda, J. N. Kutz, J. E. Cunningham, W. Y. Jan, M. Koch, and W. H. Knox, IEEE J. Sel. Top. Quantum Electron. 3, 1065 (1997).

${ }^{12}$ T. G. Euser and W. L. Vos, J. Appl. Phys. 97, 043102 (2005). 